Silvia Enciso 1,*

(1D) 0000-0001-5004-6734 Idoia Díaz-Güemes ${ }^{1}$

(i) 0000-0001-6047-2489

Blanca Fernández-Tomé 1 (1) 0000-0001-7830-3798

Belén Moreno-Naranjo'

(D) 0000-0003-2177-7049

Miguel A. Sánchez-Hurtado'

(1) 0000-0002-8731-8788

Laura Correa-Martín'

(D) 0000-0003-4470-7924

Javier Sánchez-Fernández ${ }^{2}$

(D) 0000-0002-2117-9981

Eva M. Pérez-Merino ${ }^{3}$

(D) 0000-0002-9118-1139

Jesús M. Usón-Casaús ${ }^{3}$

(1) 0000-0002-7289-7852

Francisco M. Sánchez-Margallo 4

(D) 0000-0003-2138-988X

IUnidad de Laparoscopía, Centro de Cirugía de Mínima Invasión Jesús Usón, Cáceres, Spain

${ }^{2}$ Departamento de Formación y Calidad, Centro de Cirugia de Mínima Invasión Jesús Usón, Cáceres, Spain

${ }^{3}$ Departamento de Medicina y Cirugía Animal,

Facultad de Veterinaria,

Universidad Extremadura,

Cáceres, Spain

${ }^{4}$ Dirección Cientifica,

Centro de Cirugía de Mínima Invasión Jesús Usón, Cáceres, Spain

*Corresponding authors:

Email address:

senciso@ccmijesususon.com

Accepted: 2020-06-02 Published: 2020-06-27

Additional information and declarations can be found on page 10

@) Copyright 2020 Silvia Enciso et al.

open access $\boldsymbol{\varnothing}$



Distributed under Creative Commons CC-BY 4.0

\section{Implementation of laparoscopy and thoracoscopy in small animal practice following an intensive training course}

\begin{abstract}
This study aimed to assess trainee perceptions regarding the usefulness of an intensive hands-on minimally invasive surgery (MIS) training, as well as the subsequent implementation experience. An online questionnaire was emailed to 110 small animal veterinarians who attended the course between 2007 and 2017. The questionnaire comprised three sections: general attendee information, questions pertaining to the received laparoscopic and thoracoscopic training, and finally enquiries on cumulative post-training and current MIS practice. Forty-five veterinarians answered the questionnaire. Most respondents had prior laparoscopy and/or thoracoscopy experience (95.2\%), but close to half (46.7\%), modified their clinical practice as a result of the training. Seventy-five percent of the totally inexperienced participants started practicing MIS within two years of attending the course. A large proportion of respondents indicated their need for additional training for more advanced laparoscopy (71.1\%) and thoracoscopy (77.8\%) procedures. Accordingly, the main difficulty found by respondents regarding MIS implementation was insufficient training (37.8\%). The most frequently encountered difficulty during procedures was bleeding (46.7\%), while the most feared complication was related to anesthesia (31.1\%). Ovariectomy and ovariohysterectomy were the most frequently performed minimally invasive procedures in trainee working centers. MIS training promotes the introduction of laparoscopy and thoracoscopy into clinical practice and expands the type of surgeries performed with these approaches. Nonetheless, a single intensive program does not comply with the diverse training needs of small animal practitioners.
\end{abstract}

Keywords: Laparoscopy; MIS training; Survey; Thoracoscopy

\section{Cite this as:}

Enciso S, Díaz-Cüemes I, Fernández-Tomé B, Moreno-Naranjo B, Sánchez-Hurtado MA, Correa-Martín L, Sánchez-Fernández J, Pérez-Merino EM, Usón-Casaús JM, SánchezMargallo FM. Implementation of laparoscopy and thoracoscopy in small animal practice following an intensive training course. Veterinaria México OA. 2020;7(2). doi:10.22201/ fmvz.24486760e.2020.2.857. 


\section{Introduction}

Introduced over 40 years ago in human medicine, laparoscopy offers advantages over conventional surgery, and has been considered as one of the main surgical advances in the last century. ${ }^{1}$ Laparoscopic techniques have evolved from being merely used for diagnostic or simple excising procedures, to be critical for intricate reconstructive techniques such as radical and partial nephrectomies and organ retrieval for transplant surgery, among others. ${ }^{2-4}$ Moreover, video-assisted thoracoscopic surgery (VATS) was implemented as the gold standard for the surgical treatment of operable non-small cell lung cancer during the last quarter century, its use being recently extended to more technically challenging procedures, such as lung segmentectomy. ${ }^{5-6}$

In veterinary surgery, laparoscopic and thoracoscopic procedures have been gradually incorporated and, in many cases, become the standard of care. ${ }^{7}$ As in human medicine, minimally invasive procedures diminish tissue damage, morbidity, and postoperative pain, which reduces time in the hospital and favors prompt recovery. ${ }^{8-11}$ Nevertheless, this approach is challenging for surgeons, since there is the need to adapt to a two-dimensional vision, deal with the fulcrum effect, learn to operate with decreased tactile feedback, and develop appropriate eyehand and hand-to-hand coordination. Thus, the traditional training model based on the Halstedian principle of "see-one-do-one-teach-one" is not applicable. In addition, literature regarding MIS training and its associated learning curve are scarce in veterinary medicine. ${ }^{7,13-15}$ Moreover, the implementation of MIS in clinical practice requires a substantial commitment of time and money. It demands a specialized surgical team, composed by at least the surgeon and an assistant, that operates the laparoscopic camera and, depending on the procedure, helps with an additional instrument to better expose the surgical field. ${ }^{12}$

In human medicine, several training programs have been developed, from which the "Fundamentals of Laparoscopic Surgery (FLS) simulation-based training," launched by the Society of American Gastrointestinal Endoscopic Surgeons and the American College of Surgeons in 2004, has stood out in the United States. ${ }^{16}$ The goal of this program is to enhance patient safety and care through simulation-based education, ${ }^{17}$ and residents in general surgery programs throughout the country are required to complete FLS testing prior to board certification.

Simulation-based training is widely accepted in human medicine and includes box-trainers, hybrid simulators, augmented reality simulators, and virtual reality simulators. ${ }^{18}$ Other tools, including high-fidelity models and the use of cadavers are also necessary to improve student abilities outside the clinical setting. ${ }^{14}$

Even if most veterinary school programs have basic surgical skill training, ${ }^{19}$ a concerted curriculum in surgical education does not exist. Nonetheless, veterinary students are expected to include MIS courses as part of their updated surgical training in the United States. ${ }^{20}$ Indeed, current requirements for surgical residents of the American College of Veterinary Surgeons include completion of five laparoscopic or thoracoscopic procedures that should be performed under MIS expert supervision. However, there are still not enough qualified professionals to act as supervisors, ${ }^{7}$ and training is thus reliant to some extent on incoming cases. Yet, ethical concerns arise from training on client-owned animals. ${ }^{21}$ Furthermore, expectations and evaluation of acquired surgical expertise for final year or graduate veterinary students 
include only conventional skills. $22-24$ Thus, MIS training has been established as the upcoming challenge for broadening surgical practice of veterinary students. ${ }^{14}$

Veterinarians who want to incorporate laparoscopy or thoracoscopy into their clinical setting usually opt for intensive hands-on courses to bridge the gap from conventional surgery to the minimally invasive surgical approach. This type of training can help with facing the initial complications of undertaking a new procedure, attaining the necessary surgical skills, as well as gaining confidence for treatment on client-owned animals.

Therefore, in this study, a questionnaire was designed to obtain trainee perceptions regarding usefulness and impact of a laparoscopy and thoracoscopy training program for small animal practitioners, to assess the extent to which these MIS procedures have been implemented in their clinical practice, and to identify main barriers and other issues related to the introduction of this approach.

\section{Materials and methods}

An online electronic questionnaire was designed with the SurveyMonkey application (SurveyMonkey Inc., San Mateo, California, USA) (Appendix). The online link to the questionnaire was emailed to 110 small animal veterinarians who attended our institutional intensive hands-on courses on laparoscopic and thoracoscopic surgery from 2007 to 2017. A cover letter describing the objectives of the study was also included. The comments and suggestions of veterinary MIS specialists that came as lecturers to the courses held over the three years prior to the survey $(n=12)$ were discussed to revise and construct the final version of the questionnaire. The completed questionnaire included a first section requesting basic demographic data and information on the current clinical practice of the respondent's workplace and related working resources, a second section with questions regarding general perception, usefulness and impact of the received MIS training, and a final section that inquired on general trainee experience in laparoscopy and thoracoscopy, average number of MIS procedures performed, and main complications encountered. All answers were anonymous. The SPSS 15.0 software (Chicago, IL, USA) was used to calculate and summarize the descriptive statistics.

To establish the significance of the questions within the questionnaire, a brief description of our MIS training is provided: Courses comprised 2 days of laparoscopic and 1.5 days of thoracoscopic hands-on training, centered on dry and wet laboratory practice, using synthetic models for the former, and ex vivo models and experimental animals for the latter. The course content included a 3-hour session of MIS general concept theory, hands-on practice on a simulator ( 5 hours for laparoscopy and 1 hour for thoracoscopy), and experimental animal surgeries ( 7 hours for laparoscopy and 10 hours for thoracoscopy). All participants completed every session to fulfill the course. The objectives of the theory session were to instruct participants about ergonomics and characteristics of the MIS equipment, as well as to present the most common laparoscopic and thoracoscopic techniques through clinical reports. During simulator laparoscopic training, attendants were asked to perform five tasks on a previously validated canine physical simulator. ${ }^{25}$ They began by practicing basic skills with hand-eye and hand-hand coordination tasks and cutting on inorganic tissue and continued with advanced maneuvers while 
practicing dissection and intracorporeal suturing on ex vivo porcine stomachs. Simulator thoracoscopic training included lymph node dissection and lung biopsies on ex vivo porcine cardiopulmonary systems. Once simulator skills were acquired, in vivo surgical techniques were practiced. Animals were anesthetized and tended by veterinarians to assure their welfare throughout surgeries. Laparoscopic training techniques (hepatic, renal and pancreatic biopsies, ovariectomy, nephrectomy, assisted cystoscopy and assisted gastropexy) were performed on pigs, and thoracoscopic procedures (lung biopsy, intercostal vessel dissection and occlusion, lymph node dissection, thoracic duct dissection and occlusion, and pericardiectomy) on sheep. These species were selected because of their anatomic characteristics and ease of approach and management. The surgeries were practiced in a sequence of increasing difficulty, except for the laparoscopic-assisted procedures, which were completed last to promote self-assurance and to strengthen and support the accurate execution of laparoscopic techniques. Throughout the training, participants were supervised and tutored by expert surgeons, with a mentor-to-participant ratio of $1: 2$.

Training courses were approved by our institutional Ethical Committee and complied with current European normative. ${ }^{26}$

\section{Results and discussion}

Forty-five small animal practitioners answered the questionnaire, which represented a response rate of $41 \%$. Respondents were $44.2 \pm 7.96$ years old, and worked in Spain (55.6\%), Italy (28.9\%), Portugal (8.9\%), Colombia (2.2\%), Mexico (2.2\%), and in Germany (2.2\%). Most of them were right-handed (86.7\%), and 13.3\% were ambidextrous.

Places of work included small animal clinics (57.8\%), veterinary hospitals (28.9\%), veterinary reference centers $(2.2 \%)$, ambulatory veterinary practices (6.7\%), or a setting different to the already stated options (4.4\%). Practice placement of the respondents was: owner (55.6\%), staff member (28.9\%), freelancer (13.3\%), and collaborator (2.2\%). Respondents working centers had an average of $10.23 \pm 14.8$ staff members.

The number of soft tissue surgeries (both standard and MIS procedures) performed by the respondents' workplaces within the year previous to the survey was: over 200 (44.4\%), and fewer than 50 (2.2\%). As for orthopedic surgeries, 31.1\% of the working centers did not perform orthopedic procedures, whereas $6.7 \%$ reported to have performed over 200 of these surgeries over the year preceding the survey.

Regarding the number of minimally invasive surgeries that are performed monthly within each veterinary practice, a similar trend was observed for flexible and diagnostic rigid endoscopies (such as otoscopy and rhinoscopy) (Table 1). Nearly a quarter of the working centers perform 1 to 5 laparoscopic surgeries per month (22.2\%), 26.7\% 6 to 10,31.1\% 11 to 20 surgeries, $4.4 \%$ over 20, and $15.6 \%$ none at all (Table 1). Minimally invasive thoracoscopic procedures are less frequent, with more than half of respondents declaring that no procedures were performed monthly within their places of work (Table 1). These data could imply that the incorporation of MIS has predominantly advanced soft tissue surgeons and 
Table 1. Number of monthly MIS procedures performed in the respondents' places of work

\begin{tabular}{|l|c|c|c|c|c|}
\hline \multirow{2}{*}{ MIS Procedures } & \multicolumn{7}{c|}{ Number of surgeries } \\
\cline { 2 - 6 } & None & $\mathbf{1 - 5}$ & $\mathbf{6 - 1 0}$ & $\mathbf{1 1 - 2 0}$ & $>\mathbf{2 0}$ \\
\hline Rigid endoscopy (\%) & 8.9 & 46.7 & 26.7 & 8.9 & 8.9 \\
\hline Flexible endoscopy (\%) & 8.9 & 40 & 26.7 & 17.8 & 6.7 \\
\hline Laparoscopy (\%) & 15.6 & 22.2 & 26.7 & 31.1 & 4.4 \\
\hline Thoracoscopy (\%) & 60 & 35.6 & 2.2 & 2.2 & 0 \\
\hline
\end{tabular}

Data within the body of the table indicate the percentage of veterinary practices.

endoscopists. However, as no training in additional types of surgeries were included in our course, the apparent indication of a lower number of other surgical specialty procedures, such as MIS orthopedic surgeries, cannot be ascertained.

The information collected from working centers allows for a broader picture of the type of surgical activities that the respondent is exposed to, but also posits changes that may have occurred within the practice driven by MIS course attendance. It does not however necessarily involve the participant as a surgeon, and thus a direct implication of the MIS hands-on course cannot be analyzed through these data.

Available laparoscopic infrastructure within working facilities comprises a complete laparoscopic tower (monitor, light source, camera and $\mathrm{CO}_{2}$ insufflator) in $86.7 \%$ of the clinical practices, a tower without insufflator in $8.9 \%$, an advanced bipolar device in $75.6 \%$, an ultrasound sealing device in $22.2 \%$, and surgical laser equipment in $4.4 \%$. Also, $4.4 \%$ of the veterinary practices declared not to have any laparoscopic equipment. The high percentage of veterinary centers that have complete laparoscopic towers and advanced bipolar sealing devices may relate to the fact that close to half of course attendees were apparently successful practice owners, since having close to 10 staff members within the practice could be a reflection of a busy clinic with a large clientele. Alternatively, this result could indicate that equipment investment can be afforded by small animal practices. The economic and clinical feasibility of introducing rigid endoscopy and laparoscopy to a small animal veterinary practice was evaluated in a recent study. The authors collected information over 12 months regarding performed procedures, equipment costs, training, and generated revenues, concluding that when used appropriately, incorporation of laparoscopy is possible. ${ }^{27}$ When assessing answers to this question, it should be considered that more responses could have been received from practitioners with access to laparoscopic equipment in their practices since lack of infrastructure may have influenced participants to decline answering the questionnaire.

\section{Laparoscopy and thoracoscopy training and implementation}

From the respondents that had MIS experience (46.7\%) before taking our workshop, $95.2 \%$ modified and diversified their surgical procedures as a consequence of the training received. The added MIS procedures included intracorporeal suturing, improvement in surgical approach and surgical techniques, and introduction of thoracoscopy. From the respondents without prior laparoscopy and/or thoracoscopy experience (53.3\%), a $62.5 \%$ declared to have started practicing the learned 
Table 2. Ranking of training components according to participants

\begin{tabular}{|l|c|c|c|c|}
\hline \multirow{2}{*}{ Training components } & \multicolumn{4}{|c|}{ Order of importance } \\
\cline { 2 - 5 } & $\begin{array}{c}\mathbf{1} \\
\text { (most important) }\end{array}$ & $\mathbf{2}$ & $\mathbf{3}$ & $\begin{array}{c}\mathbf{4} \\
\text { (least important) }\end{array}$ \\
\hline Theory session & 22.2 & 28.9 & 24.4 & 24.4 \\
\hline Dry lab & 11.1 & 28.9 & 33.3 & 26.7 \\
\hline Wet lab & 51.1 & 13.3 & 13.3 & 22.2 \\
\hline Mentorship & 15.6 & 33.3 & 26.7 & 24.4 \\
\hline
\end{tabular}

Data are response percentages within each category.

procedures in fewer than six months after training, a 12.5\% between 7 months and two years after training, an 8.3\% more than two years later, and a $16.7 \%$ had not performed any MIS procedure after attending the course. These data underline the importance of specialized training for MIS implementation in veterinary practice. Nonetheless, results on training impact may be biased by the fact that participants who did implement MIS after training may have been more likely to respond to the questionnaire.

Approximately one-third (37.8\%) of respondents can practice on simulators in their workplace. Runge et al. recommended that veterinarians wishing to perform laparoscopic techniques on client-owned animals have at least some experience with intracorporeal suturing using simulators or cadavers. ${ }^{28}$ Having a physical simulator in the workplace conveys an interest to train before performing MIS procedures in the clinical setting. When asked to rank the course components in order of importance, most respondents chose wet labs as the most critical, followed by theory sessions, mentorship, and finally, dry labs (Table 2).

Based on this data, $26.7 \%$ of respondents considered dry lab simulation as the least important training element. On this matter Balsa et al. found that 12\% of surveyed veterinary residents were unsure about the statement "simulation-based training improves laparoscopic skills in the operating room".7 This is surprising, since simulation training is the first step to learn laparoscopic surgical skills, its usefulness having been reported both in human and veterinary medicine. ${ }^{13,25,29}$ Certainly, Kilkenny et al. showed that simulation provides an effective platform for veterinary students (and likely novice laparoscopic surgeons), to learn laparoscopic intracorporeal suturing. ${ }^{29}$ In addition, Chen et al. compared two training curricula, the first centered on basic surgery skill training and the second based on procedures (including both physical and virtual reality simulation), and found that both improved veterinary students laparoscopic skills. ${ }^{13} \mathrm{~A}$ further study evidenced that two 50-minute physical simulation dry lab training sessions significantly enhanced the skills of inexperienced veterinarians. ${ }^{25}$ The low dry lab ranking by respondents in this study may relate to their preference to train by performing an actual procedure rather than a simple task on a simulator.

In contrast, wet lab training was considered as the most important component of the course (Table 2). Wet laboratory practice provides the most realistic environment for training outside the clinical setting, and it is thus critical for advanced procedures. Nonetheless, our experience indicates that when surgeons face experimental animal training without having the necessary surgical skills, it can be 
frustrating and counterproductive. Thus, basic surgical skills should be mastered on simulators due to both practical and ethical concerns. ${ }^{25}$

More than two-thirds (71.1\%) of the respondents declared to have a need for additional laparoscopic training, especially when advanced procedures such as cholecystectomy and urologic surgery are attempted. This requirement was even more compelling (77.8\%) regarding additional training for both basic and advanced thoracoscopic procedures. These data indicate that a single intensive MIS program is not sufficient to meet all small animal practitioner training needs, and that further instruction is required for advanced and complex procedures.

\section{Cumulative laparoscopic and thoracoscopic experience after training and current practice data}

The three main difficulties encountered by respondents when implementing MIS procedures in their clinical practice were insufficient training (37.8\%), client refusal (26.7\%), and limited time availability (24.4\%). Insufficient training was ranked over issues such as lack of equipment or manager support. In addition, even if less analgesia is required and patient recovery time is shorter in $\mathrm{MIS}^{-11}$, these procedures are more expensive than open surgeries. ${ }^{30}$ However, the economic impact was not cited as a primary limitation among respondents.

Client refusal (26.7\%) was the second main difficulty encountered by respondents for laparoscopy and thoracoscopy implementation. In a recent study, Hsueh et al. found that only $10.4 \%$ of pet owners preferred open laparotomy over MIS for female sterilization procedures, with laparoscopy and single-port laparoscopy being the first choice for $54.5 \%$ and $33.8 \%$ of the surveyed owners, respectively. ${ }^{31}$ These contrasting differences between studies may relate to geographic and socioeconomic factors, since Hsueh's study was conducted at five large university veterinary teaching hospitals in North America. Also, the question regarding encountered difficulties in our questionnaire was closed-ended, and thus it was impossible to discriminate whether pet owner refusal was related to procedure misinformation/ disinformation, costs, or other factors that may have affected the client decision.

Ovariectomy and ovariohysterectomy were the MIS procedures most performed by respondents (Tables 3-5). In general terms, this reflects that these are standard surgeries commonly performed in small animal practices, and that such procedures represent a lower level of difficulty when compared to other approaches such as thoracoscopy or additional techniques requiring intracorporeal sutures, that can be more challenging. Ovariectomy was also the most common MIS procedure reported by Jones et al., in a study where economic and clinical feasibility of introducing rigid endoscopy and laparoscopy was assessed. ${ }^{27}$ Nevertheless, other studies show that MIS implementation has expanded to include more advanced procedures, such as adrenalectomy and lung lobectomy. 11,32 This could indicate that the minimally invasive approach is gaining interest and progressing among the veterinary community.

The three most frequently encountered complications while performing MIS surgeries were bleeding (46.7\%), organ injury (related to insufflation needle or trocar insertion, 40\%), and subcutaneous emphysema (40\%). Indeed, minor bleed-

ing has been reported as the most common intraoperative complication, 8,33,34 and more extensive blood loss is one of the leading causes of emergent conversions, 
Table 3. Number of laparoscopic procedures performed by respondents over the year preceding the survey

\begin{tabular}{|l|c|c|c|c|c|c|}
\hline \multirow{2}{*}{ Type of laparoscopic procedure } & \multicolumn{7}{|c|}{ Number of surgeries } \\
\cline { 2 - 7 } & None & $\mathbf{1 - 5}$ & $\mathbf{6 - 2 0}$ & $\mathbf{2 1 - 5 0}$ & $\mathbf{5 1 - 1 0 0}$ & $>\mathbf{1 0 0}$ \\
\hline Biopsy & 20 & 33.3 & 8.9 & 17.8 & 11.1 & 8.9 \\
\hline OVE/OVH & 15.6 & 4.4 & 13.3 & 11.1 & 13.3 & 42.2 \\
\hline Laparoscopic-assisted gastropexy & 28.9 & 35.6 & 20 & 6.7 & 6.7 & 2.2 \\
\hline Laparoscopic-assisted cystoscopy & 26.7 & 33.3 & 24.4 & 8.9 & 6.7 & 0 \\
\hline Laparoscopic gastropexy & 73.3 & 13.3 & 6.7 & 6.7 & 0 & 0 \\
\hline $\begin{array}{l}\text { Advanced surgeries (cholecystectomy, } \\
\text { nephrectomy, etc.) }\end{array}$ & 62.2 & 22.2 & 11.1 & 2.2 & 2.2 & 0 \\
\hline
\end{tabular}

Data within the table are response percentages for each category. OVE, ovariectomy;

$\mathrm{OVH}$, ovariohysterectomy.

Table 4. Number of thoracoscopic procedures performed by respondents over the year preceding the survey

\begin{tabular}{|l|c|c|c|c|c|c|}
\hline \multirow{2}{*}{ Type of thoracoscopic procedure } & \multicolumn{7}{|c|}{ Number of surgeries } \\
\cline { 2 - 7 } & None & $1-5$ & $\mathbf{6 - 2 0}$ & $\mathbf{2 1 - 5 0}$ & $\mathbf{5 1 - 1 0 0}$ & $>100$ \\
\hline Biopsy & 53.3 & 33.3 & 6.7 & 6.7 & 0 & 0 \\
\hline Pericardiectomy & 51.1 & 31.1 & 11.1 & 4.4 & 2.2 & 0 \\
\hline Thoracic duct occlusion & 82.2 & 15.6 & 2.2 & 0 & 0 & 0 \\
\hline Lobectomy & 75.6 & 20 & 4.4 & 0 & 0 & 0 \\
\hline Closure of patent ductus arteriosus & 100 & 0 & 0 & 0 & 0 & 0 \\
\hline
\end{tabular}

Data are response percentages within each category.

Table 5. Number of laparoscopic and thoracoscopic surgeries performed weekly by respondents

\begin{tabular}{|l|c|c|c|c|c|}
\hline \multirow{2}{*}{\multicolumn{1}{c|}{ Procedure }} & \multicolumn{7}{c|}{ Number of surgeries } \\
\cline { 2 - 6 } & None & $\mathbf{1}$ & $\mathbf{2 - 3}$ & $\mathbf{4 - 5}$ & $>\mathbf{5}$ \\
\hline Abdominal biopsy & 42.2 & 48.9 & 6.7 & 2.2 & 0 \\
\hline OVE & 24.4 & 15.6 & 40 & 15.6 & 4.4 \\
\hline OVH & 53.3 & 24.4 & 20 & 2.2 & 0 \\
\hline Laparoscopic-assisted gastropexy & 62.2 & 33.3 & 2.2 & 0 & 2.2 \\
\hline Laparoscopic gastropexy & 84.4 & 13.3 & 2.2 & 0 & 0 \\
\hline Thoracic biopsy & 82.2 & 17.8 & 0 & 0 & 0 \\
\hline Other procedures & 68.9 & 26.7 & 2.2 & 2.2 & 0 \\
\hline
\end{tabular}

Data are response percentages within each category. OVE, ovariectomy; OVH, ovariohysterectomy. 


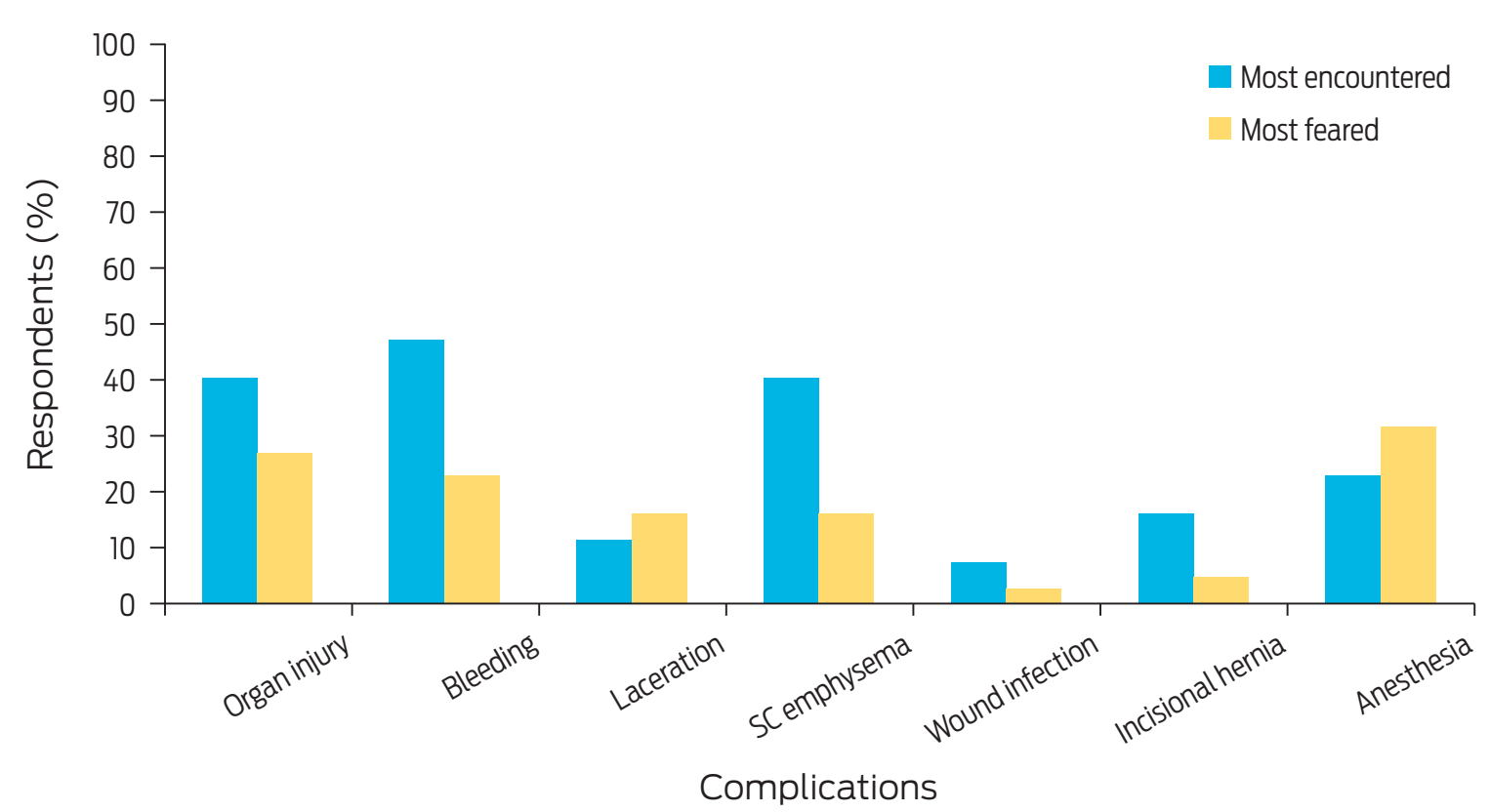

Figure 1. Most feared and more frequently encountered complications during MIS procedures. SC: subcutaneous.

where the procedure needs to be changed to an open surgery for resolution. 11,33 However, a recent study showed reduced emergent conversion rates among MIS trained human surgeons, when compared to specialists with no formal training in this area. ${ }^{35}$

Interestingly, the most feared complication when implementing the MIS procedure approach was related to anesthesia (31.1\%). This was followed by organ injury linked to insufflation needle or trocar insertion (26.7\%), and bleeding (22.2\%) (Figure 1). Special attention should be paid to underline the risk of causing pneumoperitoneum with trocar misplacement.

When performing MIS procedures, most respondents work with another veterinarian $(70.7 \%)$ or with a veterinary nurse assistant (22\%). This relates to one of the few inherent disadvantages of MIS procedures, since the surgeon must operate with a trained surgical team and an anesthesiologist. ${ }^{12}$ The relevance of additional training for anesthesiologists and nurse assistants may explain why complications related to anesthesia were the most feared by respondents.

The main limitation of this study was the low response rate which restricts our scope to appropriately determine the rate of MIS implementation after intensive training. The reasons for this low response rate are unknown, however factors such as lack of equipment or not having introduced MIS into their clinical practice may have dissuaded former course attendants to respond to the questionnaire.

The questionnaire itself also had design flaws, since requested information could have been more clearly expressed and investigated. For instance, issues such as whether needing an anesthesiologist or an assistant to implement procedures supposed an obstacle for implementation, or the possible causes for client refusal were not inquired. Thus, revision and validation of the questionnaire is necessary for future research. In addition, further studies are warranted to objectively analyze surgical performance and clinical outcomes of trainees when implementing MIS techniques. 


\section{Conclusions}

MIS training has contributed to introduce and expand laparoscopy and thoracoscopy procedures into veterinary clinical practice. However, a single intensive program is not enough to meet all training needs.

\section{Acknowledgements}

The authors gratefully acknowledge MIS trainee collaboration in this study.

\section{Conflicts of interest}

The authors declare no conflict of interest related to this report.

\section{Author contributions}

All authors contributed to the conception and design of the study, acquisition, analyses and interpretation of data, as well as manuscript preparation, critical revision, and final approval.

\section{References}

1. Minig L, Achilarre MT, Garbi A, Zanagnolo V. Minimally invasive surgery to treat gynecological cancer: conventional laparoscopy and/or robot-assisted surgery. Int J Gynecol Cancer. 2017;27(3):562-74.

2. Norris RD, Ost MC. Evolution of laparoscopy in pediatric urology. Expert Rev Med Devices. 2009;6(6):689-98.

3. Rodriguez Faba O, Boissier R, Budde K, Figueiredo A, Taylor CF, Hevia V, et al. European Association of Urology Guidelines on Renal Transplantation: Update 2018. Eur Urol Focus. 2018;4(2):208-15.

4. Lam JS, Shvarts O, Pantuck AJ. Changing concepts in the surgical management of renal cell carcinoma. Eur Urol. 2004;45(6):692-705.

5. Nakazawa S, Shimizu K, Mogi A, Kuwano H. VATS segmentectomy: past, present, and future. Gen Thorac Cardiovasc Surg. 2018;66(2):81-90.

6. Vannucci F, Gonzalez-Rivas D. Is VATS lobectomy standard of care for operable non-small cell lung cancer? Lung Cancer. 2016;100:114-9.

7. Balsa IM, Giuffrida MA, Culp WTN, Mayhew PD. Perceptions and experience of veterinary surgery residents with minimally invasive surgery simulation training. Vet Surg. 2019.

8. Devitt CM, Cox RE, Hailey JJ. Duration, complications, stress, and pain of open ovariohysterectomy versus a simple method of laparoscopic-assisted ovariohysterectomy in dogs. J Am Vet Med Assoc. 2005;227(6):921-7.

9. Mayhew PD, Brown DC. Prospective evaluation of two intracorporeally sutured prophylactic laparoscopic gastropexy techniques compared with laparoscopic-assisted gastropexy in dogs. Vet Surg. 2009;38(6):738-46.

10. Culp WT, Mayhew PD, Brown DC. The effect of laparoscopic versus open ovariectomy on postsurgical activity in small dogs. Vet Surg. 2009;38(7):811-7.

11. Mayhew PD, Culp WT, Hunt GB, Steffey MA, Mayhew KN, Fuller M, et al. Comparison of perioperative morbidity and mortality rates in dogs with noninvasive adrenocortical masses undergoing laparoscopic versus open adrenalectomy. J Am Vet Med Assoc. 2014;245(9):1028-35. 
12. Lansdowne $\mathrm{JL}$, Mehler SJ, Boure LP. Minimally invasive abdominal and thoracic surgery: principles and instrumentation. Compend Contin Educ Vet. 2012;34(5):E1.

13. Chen $C Y$, Ragle $C A$, Lencioni $R$, Fransson BA. Comparison of 2 training programs for basic laparoscopic skills and simulated surgery performance in veterinary students. Vet Surg. 2017;46(8):1187-97.

14. Fransson BA. Advances in Laparoscopic Skills Training and Management. Vet Clin North Am Small Anim Pract. 2016;46(1):1-12.

15. Runge JJ, Boston RC, Ross SB, Brown DC. Evaluation of the learning curve for a board-certified veterinary surgeon performing laparoendoscopic single-site ovariectomy in dogs. J Am Vet Med Assoc. 2014;245(7):828-35.

16. Fried GM, Feldman LS, Vassiliou MC, Fraser SA, Stanbridge D, Ghitulescu $\mathrm{G}$, et al. Proving the value of simulation in laparoscopic surgery. Ann Surg. 2004;240(3):518-25; discussion 25-8.

17. Tsuda S, Scott D, Doyle J, Jones DB. Surgical skills training and simulation. Curr Probl Surg. 2009;46(4):271-370.

18. Zendejas B, Brydges R, Hamstra SJ, Cook DA. State of the evidence on simulation-based training for laparoscopic surgery: a systematic review. Annals of surgery. 2013;257(4):586-93.

19. Cosford K, Hoessler C, Shmon C. Evaluation of a first-year veterinary surgical skills laboratory: A retrospective review. J Vet Med Educ. 2019;46(4):423-8.

20. Hill LN, Smeak DD, Lord LK. Frequency of use and proficiency in performance of surgical skills expected of entry-level veterinarians by general practitioners. J Am Vet Med Assoc. 2012;240(11):1345-54.

21. MacCormick MRA, Kilkenny JJ, Walker M, Zur Linden A, Singh A. Investigating the impact of innate dexterity skills and visuospatial aptitude on the performance of baseline laparoscopic skills in veterinary students. Vet Surg. 2017;46(8):1175-86.

22. Annandale A, Scheepers E, Fosgate GT. The effect of an ovariohysterectomy model practice on surgical times for final-year veterinary students' first live-animal ovariohysterectomies. J Vet Med Educ. 2020;47(1):44-55.

23. Cary JA, Farnsworth CH, Gay J, Carroll HS. Stakeholder expectations regarding the ability of new veterinary graduates to perform various diagnostic and surgical procedures. J Am Vet Med Assoc. 2017;251(2):172-84.

24. Bowlt KL, Murray JK, Herbert GL, Delisser P, Ford-Fennah V, Murrell J, et al. Evaluation of the expectations, learning and competencies of surgical skills by undergraduate veterinary students performing canine ovariohysterectomies. J Small Anim Pract. 2011;52(11):587-94.

25. Tapia-Araya AE, Uson-Gargallo J, Enciso S, Perez-Duarte FJ, Diaz-Guemes Martin-Portugues I, Fresno-Bermejo L, et al. Assessment of laparoscopic skills in veterinarians using a canine laparoscopic simulator. J Vet Med Educ. 2016;43(1):71-9.

26. Directive 2010/63/EU of the European Parliament, Council, on the protection of animals used for scientific purposes, September 22nd, 2010.

27. Jones K, Case JB, Evans B, Monnet E. Evaluation of the economic and clinical feasibility of introducing rigid endoscopy and laparoscopy to a small animal general practice. J Am Vet Med Assoc. 2017;250(7):795-800. 
28. Runge JJ, Mayhew P, Rawlings CA. Laparoscopic-assisted and laparoscopic prophylactic gastropexy: indications and techniques. Compend Contin Educ Vet. 2009;31(2):E2.

29. Kilkenny JJ, White K, Singh A. Evaluating veterinary student skill acquisition on a laparoscopic suturing exercise after simulation training. Vet Surg. 2019;48(S1):066-073.

30. Arulpragasam SP, Case JB, Ellison $\mathrm{GW}$. Evaluation of costs and time required for laparoscopic-assisted versus open cystotomy for urinary cystolith removal in dogs: 43 cases (2009-2012). J Am Vet Med Assoc. 2013;243(5):703-8.

31. Hsueh C, Giuffrida M, Mayhew PD, Case JB, Singh A, Monnet E, et al. Evaluation of pet owner preferences for operative sterilization techniques in female dogs within the veterinary community. Vet Surg. 2018;47(S1):015-025.

32. Bleakley S, Duncan CG, Monnet E. Thoracoscopic Lung Lobectomy for Primary Lung Tumors in 13 Dogs. Vet Surg. 2015;44(8):1029-35.

33. Buote NJ, Kovak-McClaran JR, Schold JD. Conversion from diagnostic laparoscopy to laparotomy: Risk factors and occurrence. Vet Surg. 2011;40(1):106-14.

34. Mayhew PD, Mehler SJ, Radhakrishnan A. Laparoscopic cholecystectomy for management of uncomplicated gall bladder mucocele in six dogs. Vet Surg. 2008;37(7):625-30.

35. Coffin SJ, Wrenn SM, Callas PW, Abu-Jaish W. Three decades later: investigating the rate of and risks for conversion from laparoscopic to open cholecystectomy. Surgical endoscopy. 2018;32(2):923-9. 


\section{APPENDIX \\ QUESTIONNAIRE}

\section{Section 1. Demographic data and basic information}

Year of birth:

Country:

Laterality:

Right-handed

Left-handed

Ambidextrous

Veterinary practice:

Small animal

Large animal

Exotic animal

Other(specify please):

\section{Workplace:}

Veterinary clinic

Veterinary hospital

Ambulatory practice

Other (specify please):

Number of veterinarians in your workplace:

Position:

Owner

Staff member

Freelancer

Other (specify please):

Total number of surgeries performed last year:

\begin{tabular}{|c|c|c|c|c|c|c|}
\hline & $<50$ & $51-100$ & $101-150$ & $151-200$ & $>200$ & NA \\
\hline Orthopedic surgery & & & & & & \\
\hline Soft tissue & & & & & & \\
\hline
\end{tabular}

Number of surgeries performed monthly in your workplace:

\begin{tabular}{|l|l|l|l|l|l|}
\hline Flexible endoscopy & & & $\mathbf{1 1 - 2 0}$ & $\mathbf{2 0}$ \\
\hline $\begin{array}{l}\text { Diagnosticrigidendoscopy } \\
\text { (rhinoscopy, otoscopy, etc.) }\end{array}$ & & & & & \\
\hline Laparoscopic surgery & & & & & \\
\hline Thoracoscopic surgery & & & & & \\
\hline
\end{tabular}


In your workplace you have available:

Complete laparoscopic tower (monitor, light source, camera and $\mathrm{CO} 2$ insufflator) Tower without insufflator Advanced bipolar device Ultrasound sealing device Other (specify please):

\section{Section 2. Laparoscopy and thoracoscopy training}

Did you perform laparoscopy/thoracoscopy before receiving the training? Yes

No

Comments:

If your response was AFFIRMATIVE, have you modified your practice as a consequence of the training received?

Yes

No

If your response was affirmative, what have you changed?

If your response was NEGATIVE, how long has it take after the training until the implantation of laparoscopy/thoracoscopy in your workplace?

$<6$ months

7 months-2 years

$>2$ years

Other (specify please):

What training elements do you consider more important to favor the implantation of laparoscopy/thoracoscopy? Order from 1 to 4 , being 1 the most important Theory

Dry lab

Wet lab

Mentorship

Have you the possibility to practice on a simulator in your workplace?

Yes

No

Would you need additional training in any area for laparoscopic implementation?

Y Yes

No

If your response was AFFIRMATIVE, in which area?

Would you need additional training in any area for thoracoscopic implementation?

Yes

No

If your response was AFFIRMATIVE, in which area? 
Section 3. Cumulative laparoscopy and thoracoscopy experience after training and current practice data

What difficulties have you encountered for laparoscopy and thoracoscopy implementation in your workplace? Mark the 3 most important

Lack of equipment

Lack of support from the management

Lack of appropriate patients

Clients' refusal

Little availability of time

Lack of a trained anesthetist

Insufficient training

Other (specify please):

Laparoscopic surgery (total no. of procedures performed last year):

\begin{tabular}{|l|l|l|l|l|l|l|}
\hline \multicolumn{2}{|c|}{ None } & $1-5$ & $\mathbf{6 1 - 2 0}$ & & \\
\hline Biopsy & & & & & & \\
\hline OVE/OVH & & & & & & \\
\hline $\begin{array}{l}\text { Laparoscopic-assisted } \\
\text { gastropexy }\end{array}$ & & & & & & \\
\hline $\begin{array}{l}\text { Laparoscopic-assisted } \\
\text { cystoscopy }\end{array}$ & & & & & & \\
\hline Laparoscopic gastropexy & & & & & & \\
\hline $\begin{array}{l}\text { Advanced procedures } \\
\text { (cholecystectomy, } \\
\text { nephrectomy, etc.) }\end{array}$ & & & & & & \\
\hline
\end{tabular}

Thoracoscopic surgery (total no. of procedures performed last year):

\begin{tabular}{|l|l|l|l|l|l|l|}
\hline \multicolumn{9}{|c|}{ None } & $1-5$ & $61-100$ & $>100$ \\
\hline Biopsy & & & & & & \\
\hline Pericardiectomy & & & & & & \\
\hline Thoracic duct occlusion & & & & & & \\
\hline Lobectomy & & & & & & \\
\hline $\begin{array}{l}\text { Closure of patent ductus } \\
\text { arteriosus }\end{array}$ & & & & & & \\
\hline
\end{tabular}

Number of procedures performed weekly:

\begin{tabular}{|l|l|l|l|l|l|}
\hline \multicolumn{2}{|c|}{ None } & & & & \\
\hline Abdominal biopsy & & & & & \\
\hline OVE & & & & & \\
\hline OVH & & & & & \\
\hline Laparoscopic-assisted gastropexy & & & & & \\
\hline Laparoscopic gastropexy & & & & & \\
\hline Thoracic biopsy & & & & & \\
\hline Other procedures & & & & \\
\hline
\end{tabular}


Mark the 3 most frequent complications that you have encountered:

Organ injury during insufflation needle or trocar insertion

Bleeding

Lacerations to adjacent structures

Emphysema

Wound infection

Incisional hernia

Anesthetic related

Other (specify please):

Mark the 3 complications that you most feared when implanting laparoscopy/thoracoscopy?

__ Organ injury during insufflation needle or trocar insertion

Bleeding

Lacerations to adjacent structures

Emphysema

Wound infection

Incisional hernia

Anesthetic related

Other (specify please):

Who do you work with to perform laparoscopy/thoracoscopy?

Veterinarian

Assistant/Nurse

Nobody

Other (specify please): 